\title{
LXII. On the untenableness of the received theory of Newton's rings
}

\section{E. Wilde}

To cite this article: E. Wilde (1850) LXII. On the untenableness of the received theory of Newton's rings, Philosophical Magazine Series 3, 37:252, 451-462, DOI: $10.1080 / 14786445008646653$

To link to this article: http://dx.doi.org/10.1080/14786445008646653

曲 Published online: 30 Apr 2009.

Submit your article to this journal $₫$

Џ Article views: 3

Q View related articles $₫$ 
need scarcely be taken into consideration as any set-off against a principle promising a gain of forty to one.

The following additional demonstration of this principle having been submitted to Mr. Walker, he requested that it may be added as corroborative of his views.

If the French experiment of the wedge form, represented by fig. 3, were made only one foot deep, by two feet wide at the base, that half of the prow below the centre line would correctly represent the plane $\mathrm{AB}$, fig. 4 ; and if both were moved horizontally with the velocity of ten feet per second, as from $A B$ to $\mathrm{CD}$, the plane $\mathrm{AB}$ would just meet half the resistance of the prow.

Experiment has shown that the two square feet of base to the prow would, if the prow were absent, at that velocity receive 100 pounds resistance each, $=200$ pounds; and that, with the prow, the resistance would be $\frac{4}{10}$ ths of this, or 80 pounds. Thus one-half, or 40 pounds, would be the horizontal resistance of the plane $A B$, moving ten feet per second, which is equivalent to 400 pounds moved one foot per second.

As the resistance varies as the squares of the velocities, if the plane $\mathrm{AB}$ were placed horizontally, as represented by $\mathrm{AE}$, and then depressed in one second to $\mathrm{DB}$ (being one foot), each of the ten square feet would only receive one pound of resistance, and the whole would be ten pounds moved one foot per second, which is just one-fortieth part of the power required in the former case. Q. E. D.

G. C.

November 4, 1850.

LXII. On the Untenableness of the received Theory of Newton's Rings. By E. WILDE*

A DESIRE to investigate the colours of thin plates more A. closely induced me to have an instrument prepared, by which I am enabled actually to measure the diameters of the coloured rings of Newton to the ten-thousandth part of an English inch, and to attain by estimation an accuracy of one hundred-thousandth of an inch; the same instrument determines the approximation of the glasses to the millionth of an inch. It is therefore capable of a greater degree of exactness than even the spherometer of Biot. I will name it a gyreidometer.

Some years ago an instrument for the exhibition of Newton's rings was invented by Jericau in Sweden; it was named a gyreidoscope by its inventor. The instrument however

* Translated from Poggendorff's Annalen, vol. lxxx. p. 407, July 1850. $2 \mathrm{G} 2$ 
could not be used for the purposes of measurement, and hence I found myself compelled to resort to other principles in the construction of my own.

In the description which he has given of his gyreidoscope, Jericau dissents from the theory of Newton's rings, which up to the present time has been accepted; inasmuch as experiment has taught him, that, by pressing the glasses very strongly together, the centre, instead of appearing dark, as asserted by Newton, actually becomes bright. He endeavoured to explain the matter by referring it to the repeated diffusion of colours within a circle. I must however confess that I am unable to attach any clear meaning to his explanation.

Prompted, however, by this remark of Jericau's, I was induced to' undertake a closer investigation of the subject as soon as I found the gyreidometer in my possession. I have not been able to corrohorate the statement, that by pressing the glasses very strongly together the centre becomes bright. I have found that the first result of the pressure is a dark central spot, which is formed at the summit of the convex lens; but by increasing the compression and bringing the glasses more closely into contact, the black spot expanded into a circle with a bright centre; in the middle of this bright patch a dark spot again appeared as the pressure was increased, and it thus continued changing according as the length of the paths of the respective rays reflected at the upper and lower limit of the layer of air differed from each other by an odd or even number of semi-undulations, until finally a constant dark central spot was obtained, which it was impossible to supersede even when the glasses were so forcibly pressed, that the upper one, which was a quarter of an inch in thickness, actually bent under the force. As the spot increased in size, the surrounding curves diverged more and more from the circular form. By ordinary daylight, as might be expected, the change from dark to bright, which accompanied the approach of the glasses, could not be perceived; a variety of colours exhibited themselves one after the other, until, finally, upon very strong pressure, the dark central spot was obtained. A small number only of rings were visible when viewed with common light; but with homogeneous light the field of view of the microscope appeared covered with many hundred curves, alternately dark and bright.

But although I find myself thus unable to coincide with the assertion of Jericau, a careful examination of the rings of Newton with the gyreidometer has furnished me with other grounds of dissent from the generally received theory which 
has been deduced from the theory of undulation by Young, Fresnel, Poisson, Herschel, Airy, and others. My grounds of dissent are as follow:-

1. It is theoretically impossible that a reversion of the æther-vibrations, reflected at the lower limit of the one glass as compared with those reflected at the upper limit of the other, can take place when there is no layer of air between the glasses.

2. If, in order to preserve the received theory, a layer of air so thin that its depth is very small even in comparison with the length of an undulation, be assumed to exist at the point where the dark spot commences to show itself, this could not account for the continuance of the spot after the glasses have been pressed so strongly together that no air can possibly exist between them. This assumption therefore does not help us ; the central spot cannot be referred to the interference of the rays reflected at the respective limits of the glasses, though this is the explanation which has heretofore been accepted.

3. In reflected daylight the central spot appears a deep black. Its origin, therefore, even should we grant the existence of an intermediate air-layer, can by no means be referred to the principle of interference; for it is well known, that, owing to the difference in the lengths of the respective undulations, the interference of rays compounded of all colours must result in a tinting more or less vivid. Since therefore the central spot cannot be referred to the existence of a thin layer of air, nor for the reason first assigned be referred to the principle of interference, we are driven to seek a different explanation of the phænomenon from that heretofore given.

4. Because, with homogeneous light, if the distance of the glasses from ench other be equal to nought, and a reversion of the vibrations consequently impossible, the phases of the rays reflected from the two glasses at the point of contact must correspond, and a dark spot cannot be the consequence.

5. Because, in the case under consideration, the phænomena of colour which are exhibited in all analogous cases, among which may be reckoned the figures due to diffiration, are entirely absent.

From all these grounds it follows, that the middle of the ringsystem, when the distance between the glasses is nought, cannot appear dark in reflected light; but that, in direct opposition to all assertions heretofore made, it onght to appear bright.

The shortest proof for the so-called law of Young, I find in Fresnel's expression for the velocity of oscillation of a reflected ray polarized in the plane of incidence, and of a reflected ray 
polarized perpendicular to the plane of incidence; the former (that is, of the ray in which the oscillations are at right angles to the plane of incidence and to the direction of the ray) has the following value :-

$$
-\frac{\sin (i-r)}{\sin (i+r)}, \text {. . . . . . }
$$

and the latter (that is, of the ray in which the oscillations are parallel to the plane of incidence and perpendicular to the direction of the ray) the value*-

$$
\frac{\tan (i-r)}{\tan (i+r)} \text {. . . . . . }
$$

At the upper limit of the layer of air, the angle of incidence $i$ is smaller than the angle of refraction $r$, the passage in this case being from glass to air ; the expression (1.) is therefore positive. At the lower limit of the air-layer, on the contrary, the angle $i$ is greater than $r$, the passage in this case being from air to glass; and the same expression is consequently negative. In the same way the expression (2.) changes its sign according as it refers to the one or the other limit of the air-layer; at the upper limit it is negative, and at the lower limit it is positive. A change of sign, however, in the velocity of oscillation is always accompanied by a reversion of the direction of oscillation. Hence, when a natural (unpolarized) ray, which as regards intensity may be considered as composed of the two polarized rays mentioned above, is reflected at the lower boundary of the layer of air, a reversion of the rether oscillations, as compared with their direction after reflexion from the upper boundary, must take place, which reversion, as regards the intensity of the reflected light, has the same effect as if the difference of the paths traversed by both rays was half an undulation (or in general an odd number of semi-undulations) greater or less than it really is; this however under the sole condition that a layer of air exists between the two glasses. If therefore a difference of half an undulation has been heretofore assumed at the point where no air exists between the glasses, the said assumption has been made in direct opposition to the theory of undulation.

If, on account of the reversion of the oscillation, I assume the difference of the paths traversed by the interfering homogeneous rays half an undulation $\lambda$ (or an odd number of half undulations) greater or less than it really is, then the simplest expression for the intensity of the reflected light $I$ find to be

$$
\mathrm{J}=4 a \sin ^{2} 2 \pi \frac{d \cos r}{\lambda}, \text {. . . . . }
$$

* Poggendorff's Annalen, vol. xxï. p. 90. 
where $a$ represents the quantity of light reflected at the upper or lower boundary of the layer, $d$ the depth of the layer, and $r$ the angle of refraction from glass to air. For the transmitted light, on the contrary, where no reversion of the vibration takes place, the intensity is

$$
\mathrm{J}^{\prime}=1-4 a \sin ^{2} 2 \pi \frac{d \cos r}{\lambda}, . \quad . \quad .
$$

so that the intensities are complementary, the sum of both being the intensity 1 of the incidental light.

For reflected light, we obtain from (3.) the maxima of intensity for

$$
d=\frac{\lambda}{4 \cos r},=\frac{3 \lambda}{4 \cos r},=\frac{5 \lambda}{4 \cos r},=\frac{7 \lambda}{4 \cos r}, \ldots .
$$

and the minima of intensity for

$$
d=0,=\frac{2 \lambda}{4 \cos r},=\frac{4 \lambda}{4 \cos r},=\frac{6 \lambda}{4 \cos r}, \ldots \ldots
$$

because for the first series $J=4 a$, and for the second $J=0$. For the transmitted light, we obtain from (4.)

the maxima for

$$
d=0,=\frac{2 \lambda}{4 \cos r},=\frac{4 \lambda}{4 \cos r},=\frac{6 \lambda}{4 \cos r}, \ldots \ldots
$$

and the minima for

$$
d=\frac{\lambda}{4 \cos r},=\frac{3 \lambda}{4 \cos r},=\frac{5 \lambda}{4 \cos r},=\frac{7 \lambda}{4 \cos r}, \ldots .
$$

because for the first series $J^{\prime}=1$, and for the second series $\mathbf{J}^{\prime}=1-4 a$. Now as the diameters or radii of the coloured rings are proportional to the square roots of the depths of the layers of air, the diameters or radii of the maxima in the case of reflected light bear to each other the ratio $\sqrt{ } 1: \sqrt{ } 3: \sqrt{ } 5 \ldots$; and those of the minima the ratio $\sqrt{ } 0: \sqrt{ } 2: \sqrt{ } 4 \ldots$. With transmitted light, on the contrary, the ratios of the maxima will be $\sqrt{ } 0: \sqrt{ } 2: \sqrt{ } 4 \ldots$, and those of the minima $\sqrt{ } 1$ : $\sqrt{ } 3: \sqrt{ } 5 \ldots \ldots$

This is the manner in which Newton first expressed the law, and in the same manner it has heretofore been deduced from the undulation theory. It is however so far from agreeing with the same, that the law, to be rendered applicable to the centre of the ring-system, would require entire reversion.

The rings nearest the centre begin to lose their circular shape, and to assume one more or less elliptical, immediately before the appearance of the constant dark spot, and while the centre is still bright. There can be no doubt that actual 
contact has taken place, and that the distance between the glasses is already $=0$ when the shape of the rings has begun thus to alter, inasmuch as this alteration manifestly intimates an actual pressure of the glasses. Then, however, the first bright ring must appear as the uninterrupted continuation of the bright central patch, because the first dark ring is not formed until for $\cos r=\cos 0^{\circ}=1$ the depth $d$ of the layer of air is $\frac{2 \lambda}{4}$, the difference of the paths of the interfering rays (taking into account the journey forward and back through the airlayer and the reversion of the vibration) being at this place $=2 \frac{2 \lambda}{4}+\frac{\lambda}{2}=\frac{3 \lambda}{2}$, and the light consequently destroyed. When this is the case, we have with reflected light, the maxima for

$$
d=0 \text { and } \frac{\lambda}{4 \cos r},=\frac{3 \lambda}{4 \cos r},=\frac{5 \lambda}{4 \cos r},=\frac{7 \lambda}{4 \cos r}, \ldots .
$$

and the minima for

$$
d=\frac{2 \lambda}{4 \cos r},=\frac{4 \lambda}{4 \cos r},=\frac{6 \lambda}{4 \cos r},=\frac{8 \lambda}{4 \cos r} \ldots \ldots
$$

With transmitted light, on the contrary, the maxima for

$$
d=\frac{2 \lambda}{4 \cos r},=\frac{4 \lambda}{4 \cos r},=\frac{6 \lambda}{4 \cos r},=\frac{8 \lambda}{4 \cos r}, \ldots .
$$

and the minima for

$$
d=0 \text { and } \frac{\lambda}{4 \cos r},=\frac{3 \lambda}{4 \cos r},=\frac{5 \lambda}{4 \cos r},=\frac{7 \lambda}{4 \cos r}, \ldots \ldots
$$

from which it follows, that with reflected light the radii of the successive bright rings are in the ratio of $\sqrt{ } 1: \sqrt{ } 3: \sqrt{ } 5 \ldots \ldots$, and those of the dark rings in the ratio of $\sqrt{ } 2: \sqrt{ } 4: \sqrt{ } 6 \ldots \ldots$. With transmitted light, however, the radii of the bright rings are in the ratio $\sqrt{ } 2: \sqrt{ } 4: \sqrt{ } 6 \ldots$, and of the dark rings in the ratio $\sqrt{ } 1: \sqrt{ } 3: \sqrt{ } 5 \ldots$; the middle of the system, up to the points where the layer of air reaches a depth of $d=\frac{2 \lambda}{4}$, appearing at the same time in maxinum with reflected light, and in minimum with transmitted light.

The measurements I have made with the gyreidometer, in which were placed a plane glass and a convex glass of 360 inches Eng. radius, are my security against error here. When using reflected light, the glasses were pressed together until the circular form of the rings began to alter, the middle of 
the system however being still bright, with the homogeneous light of a lamp of alcohol and chloride of sodium* for the angle of incidence $39^{\circ} 4 \mathrm{l}^{\prime}$ (the angle of refraction $r$ from glass to air), I found, by taking in each case the mean of repeated measurements, the radii to be-

\begin{tabular}{|c|c|c|}
\hline \multicolumn{3}{|c|}{$\begin{array}{l}\text { Reflected Light. } \\
\text { Radii of the }\end{array}$} \\
\hline first, & $\begin{array}{lc}\text { second, } & \text { third, } \\
0 \cdot 1244^{\prime \prime} \text { Engl. } & 0 \cdot 1602^{\prime \prime}\end{array}$ & $\begin{array}{c}\text { fourth bright ring. } \\
0.1900^{\prime \prime}\end{array}$ \\
\hline $\begin{array}{l}\text { first, } \\
0 \cdot 1021^{\prime \prime} \text { Engl. }\end{array}$ & $\begin{array}{l}\text { second, } \\
0^{\prime} 1439^{\prime \prime}\end{array}$ & $\begin{array}{c}\text { fourth dark ring. } \\
\qquad 0.2040^{\prime \prime}\end{array}$ \\
\hline
\end{tabular}

These values however need a sensible correction, inasmuch as the radii of the rings, on account of the refraction of the upper plate, a quarter of an inch thick, appear snaller than they really are at the upper limit of the air-layer. The mean exponent of refraction necessary for the ascertaining of these corrections, I obtained according to the method of Prechtl t, from a series of ten measurements. The microscopic lens being $2 \cdot 91$ English inches distant, the following corrections are obtained:-

Reflected Light.

Corrections for the

$\begin{array}{cccc}\text { first, } & \text { second, } & \text { third, fourth bright ring. } \\ \ldots & 0.0070^{\prime \prime} & 0.0091^{\prime \prime} & 0.0106^{\prime \prime} \\ \text { first, } & \text { second, } & \text { third, fourth dark ring. } \\ 0.0056^{\prime \prime} & 0.0081^{\prime \prime} & 0.0099^{\prime \prime} & 0.0113^{\prime \prime}\end{array}$

These corrections being applied to the radii found above, and the radius of the first bright ring being calculated from the second by means of the proportion 1: $\sqrt{ } 3$, we obtain, finally,-

\section{Reflected Light.}

Radii of the

$\begin{array}{cccc}\text { first, } & \text { second, } & \text { third, fourth bright ring. } \\ 0.0758^{\prime \prime} & 0.1314^{\prime \prime} & 0.1693^{\prime \prime} & 0.2006^{\prime \prime} \\ \text { first, } & \text { second, } & \text { third, } & \text { fourth dark ring. } \\ 0.1077^{\prime \prime} & 0 \cdot 1520^{\prime \prime} & 0 \cdot 1864^{\prime \prime} & 0.2153^{\prime \prime}\end{array}$

* This light is not completely homogeneous, but a mixture of yellow and violet, as I found by decomposition with a prism; so that it deserves to be called orange rather than yellow. It is however sufficiently homogeneous for the purpose to which it has been here applied.

$\uparrow$ Practical Dioptrics. Vienna, 1828, p. 127. 
which values are so exact, that, when set down in the form of a proportion with the square roots found above, the product of the extremes agrees with the product of the means to at least four places of decimals. 'Thus, for example, for the first and third dark rings, the following proportion ought to hold good, $0 \cdot 1077^{2}: 0 \cdot 1864^{2}=0 \cdot 01159: 0 \cdot 03474=2: 6=1: 3$, which it actually does, the product of the extremes agreeing with the product of the means to four places, viz. 0.0347.

The laws deduced from the undulation theory for the reflected light are thus confirmed by experiment, without its being necessary to press the glasses so strongly together as to cause the dark central spot to exhibit itself; and this is the point which it was my object to prove.

Another consideration which supports the truth of my assertion is, that by all analogous phænomena of colour where, when homogeneous light is used, an alternation of dark and bright is observed, the middle being bright, the first minimum occurs where the paths traversed by the interfering rays differ by at lenst an entire undulation. Thus, for example, in the alternation which occurs when light, after having passed through a narrow opening, is received upon an opake screen, the middle, where the difference of path is nothing, is always bright, its intensity being $=1$. 'The space however remains also bright when the difference amounts to half an undulation, its intensity at this point being $0 \cdot 4053$, and it is not until the difference amounts to an entire undulation that the first minimum occurs*. The resemblance is closer still when the diffraction is caused by the rays passing through a small circular opening, in which case the middle is also bright, and the first dark ring occurs where the difference of the paths traversed by the rays is $1 \cdot 220 \lambda+$. It is, on this account, impossible to determine the difference of path corresponding to the first maximum ring, which appears as a continuation of the bright centre; this difference must be obtained from the expression of intensity (3); and for the same reason I have been unable to ascertain by measurement the radius of the first bright ring, being obliged also to resort to the expression (9.) to obtain it. Notwithstanding, however, that in all these related phænomena, where the centre is bright, the first minimum does not occur where the difference is half an undulation, still up to the present time it has been assumed, in the case of transmitted light, that the centre of Newton's ring-system is bright, and that the first dark ring nevertheless occurs where the depth of the layer of air is one-fourth of an undulation, that is to say, where the difference of paths amounts to half an un-

- Poggendorff's Annalen, vol, lxxix, p. 206.

+ Ibid. p. 224. 
dulation, for in this case the vibrations are not reversed. No analogy therefore exists between the theory of Newton's rings heretofore received, and that which explains other phænomena of a similar nature.

The error is evidently due to the circumstance, that, trusting to the accuracy of Newton's observations, the formula of intensity (3.), which applies solely to the case where a reversion of the æether vibrations may be assumed, has been extended to the case where the distance heing 0 , no reversion is possible. It may be remarked, further, that the depth 0 has never been introduced into the proportion, nor could it be so introduced, as a fourth proportional infinitely great would be the consequence. It is thus easy to conceive how, neglecting to test the original observations of Newton, the same error might be repeated through centuries.

With regard, finally, to the origin of the central spot, which by daylight appears black, but by homogeneous light, on account of the dull background, does not appear darker than the rings caused by interference, my opinion coincides with that of Jericau, that the spot is caused by transmitted light in the same manner as the spot upon a mirror when a little of the metallic coating is removed from behind. The part from which the coating has been removed appears dark in comparison with the surrounding bright reflecting surface. I however agree with this notion, not as a probable conjecture, but as an undeniable fact. The grounds of this assertion are the following :- -

Let DEC represent a glass prism, $O$ the place of the eye, and $\mathrm{GH}$ a line parallel to the ends. A refraction of red light is first possible at $t$ when the angle $\mathrm{O} t \mathrm{G}$ is $=49^{\circ} 2^{\prime}$, or the angle of incidence at $t=48^{\circ} 58^{\prime}$; and a refraction of the violet in $q$ first occurs when the angle $\mathrm{O} q \mathrm{G}$ $=50^{\circ} 7^{\prime}$, or the angle of incidence $=39^{\circ} 53^{\prime}$. Let $p$ be the place at which the middle rays are refracted.

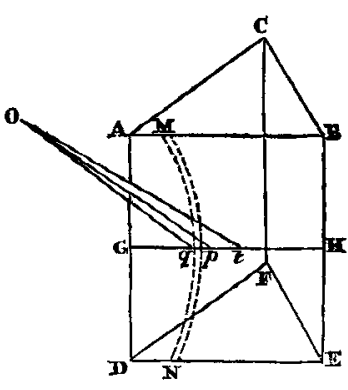
Between $t$ and $\mathrm{BE}$ no ray will be broken at the base $\mathrm{DB}$, and hence in this portion a total reflexion will take place. Between $t$ and $p$, red, orange and yellow rays can pass through; and, on account of their absence, the light reflected from this portion will be diminished in intensity. Between $p$ and $q$ all the other rays, blue and violet excepted, are allowed to pass ; here therefore the intensity of the reflected light will be still less, while in the vicinity of $q$, where the blue and violet have 
not yet found a passage, the bluish bow MN is observed, which Newton was the first to notice and explain. In the portion of the base between $\mathrm{MN}$ and $\mathrm{AD}$, where all the rays are refracted, the reflected light is for this reason very dull.

Having laid the basis DB of the prism upon the summit of a very flat convex lens, I pressed both glasses forcibly together, and inclined the eve towards the base, so that I could see the place where the glasses touched between $q$ and $\mathrm{AD}$. Here were exhibited a considerable number of rings, such as those observed in daylight with two very flat convex lenses; a complete central spot of deep black was visible at the same time. The eye being bent still lower while the glasses remained unaltered, I observed the place of contact between $q$ and $p$; the central spot appeared no longer black, but a most vivid green. When the eye was inclined still more, colours of the brightest yellow, orange, and at last red, were exhibited successively, until finally, when the eye was sunk still lower, so that the place of contact came into the vicinity of total reflexion, the deep black spot again appeared complete as before.

This experiment is decisive. It proves that the hue of the central spot depends upon transmitted light, and for ever sets aside the possibility of the assumption, that the origin of the spot is to be sought in interference. The colours thus obtained are perfectly homogeneous; and hence, if the idea of interference could in the least be admitted, the central spot must remain invariably black. 'This however is not the case. The colours green, yellow, orange, which the central spot assumes, are as vivid and pure as those obtained from the best fintglass prisms, which is especially evident when the glasses are laid upon black paper and the light prevented from falling upon the lens itself. Of this I convinced myself by placing an opake screen on that portion of the lens turned towards the light which was uncovered by the prism, and thus holding back those rays which could fall upon the base from below. Had the lens a stronger convexity, the colours of the central spot, as may be readily conceived, would not be sufficiently separated.

Although this experiment renders further proof of the correctness of my assertion superfluous, I will nevertheless introduce a few others which have furnished me with the same results.

Upon the side of the prism turned towards the light, I laid a piece of black paper, so that light could only be received through the lens from below. The lens and prism being pressed more forcibly together, the place of contact, when observed 
through the side of the prism turned towards the room, appeared like an uninterrupted and very bright opening passing through both glasses, while the surrounding portion of the base of the prism was dull and dim. This experiment proves the uninterrupted passage of the rays through the centre where the light falls from above upon the uncovered side of the prism.

When two lenses placed together were laid upon white paper, the hue of the central spot appeared less intense than when black or merely dark-coloured paper was used, and the spot was not at all visible except when the light was obliquely reflected. At smaller angles of incidence it vanished totally, and appeared as a white circle when I looked vertically down upon the place of contact of the glasses, the ring-system being visible at the same time. If, however, the darkness of the central spot was caused, as heretofore supposed, by interference, by a self-destruction of the light, the spot must continue dark, even when the glasses are laid upon a white ground, under all possible angles of incidence, and the light proceeding upwards from the paper could not cause the place of contact to appear white, as it actually does.

Having united the summits of two convex lenses with $\mathrm{Ca}$ nadian balsam, I laid them on a black or dark-coloured surface, and observed, by all incidences of the rays, that the place on which the balsan lay exhibited the same deep black as when two glasses are pressed together in daylight. When, however, the lenses were laid upon white paper, the black, as in the case of the pressed glasses, was less intense, and the spot also disappeared when small angles of incidence were used. Although the continuity of the glasses in this case was only partially effected by the balsam, still every experiment made with the lenses thus united pronounced decisively that the darkness of the central spot is to be referred exclusively to the free transmission of the light at the place of contact.

In the first experiment, it is observed that in the neighbourhood of total reflexion, on the base of the prism, the central spot is not surrounded by rings. Rings cannot show themselves, because at the place where they ought to appear no rays are transmitted which after reflexion from the lens could interfere with those reflected from the base. The central spot, however, appears black, because as no air exists between the glasses at the point of contact, no total reflexion is here possible, the light being permitted to pass on. For were we to assume the existence of a layer of air at the place of contact, the light must undergo total reflexion at this point also, and hence the central spot must appear as bright as the surround- 
ing portion. Since, therefore, the presence of a layer of air cannot be here assumed, the darkness of the central spoteven setting aside the consideration that black can never result from the interference of heterogeneous rays-cannot arise from interference, because the latter without an air-layer is impossible.

The fuller development of the views expressed in the foregoing pages I reserve to a future memoir. In submitting my divergence from received opinions to the judgement of those who understand the subject, my sole object is to bring all optical action, no matter of what particular kind, into coincidence with the theory of undulation; so that the harmony of our thoughts with the divine order of Nature, at least in this department of science, shall remain undisturbed.

LXIII. Note to a former paper "On an alleged proof of the "Method of Least Squares." " By R. L. ElLIs, late Fellow of Trinity College, Cambridge.

To the Editors of the Philosophical Magazine and Journal.

Gentlemen,

A LLOW me to correct an error in my letter to Professor A Forbes, published in your last Number. The Edinburgh reviewer, on whose proof of the method of least squares I was commenting, says that the most probable position of the wafer is the centre of gravity of the shot-marks; of course on the supposition that in this, as in all other cases, the probability of a deviation or error $r$ is equal or proportional to a certain constant base raised to the power -1 .

Now, admitting this supposition to be true, the centre of gravity is not the most probable position of the wafer. But, on the contrary, if the function mentioned at the close of $m y$ former communication, viz. $2 h^{2} e^{-h^{2} r^{2}} r d r$, expresses the probability of an error $r$, then the centre of gravity is the most probable position. I thus not only omitted to notice that the reviewer's conclusion would not follow from his own hypothesis, but by this omission was led to introduce an error of my own,

It is unnecessary to trouble you with a proof of what $I$ have now said, as the matter does not affect the general question.

I am, Gentlemen,

Brighton, Nov. 7 .

Your obedient Servant,

R. L. ELLis. 